\title{
Normal values of $M$ mode echocardiographic measurements of more than 2000 healthy infants and children in central Europe
}

\author{
C Kampmann, C M Wiethoff, A Wenzel, G Stolz, M Betancor, C-F Wippermann, \\ R-G Huth, P Habermehl, M Knuf, T Emschermann, H Stopfkuchen
}

\begin{abstract}
Objective-To obtain normal $M$ mode (one dimensional) echocardiographic values in a substantial sample of normal infants and children.

Design-Data were obtained over three years from a single centre in central Europe.

Patients-2036 healthy infants and children aged one day to 18 years.

Methods-In line with recommendations for standardising measurements from $M$ mode echocardiograms, and using digital echocardiographic equipment, measurements were obtained of the following: right ventricular anterior wall thickness at end diastole, right ventricular end diastolic dimension, thickness of interventricular septum at end diastole and end systole, thickness of posterior wall of the left ventricle at end diastole and end systole, left ventricular dimension at end diastole and end systole, pulmonary and aortic valve diameter, and left atrial dimension.

Results-Measurements are presented graphically on centile charts with respect to body surface area, and as tables with mean and 2 SD values for newborns in relation to body weight, and for infants and children in relation to body surface area. Best fitting regression equations are given for each measured variable, using the 50th centile values.

Conclusion-In comparison with previously published normal values, the presented charts and tables make it possible to judge echocardiographic measurements of a particular patient as normal or abnormal.

(Heart 2000;83:667-672)
\end{abstract}

Johannes Gutenberg University, Department of

Paediatric Cardiology, Langenbeckstrasse 1, D-55101 Mainz,

Germany

C Kampmann

C M Wiethoff

A Wenzel

G Stolz

$M$ Betancor

C-F Wippermann

R-G Huth

P Habermehl

M Knuf

T Emschermann

H Stopfkuchen

Correspondence to:

Dr Kampmann

email:

christoph.kampmann@

uni-mainz.de

Accepted 1 March 2000
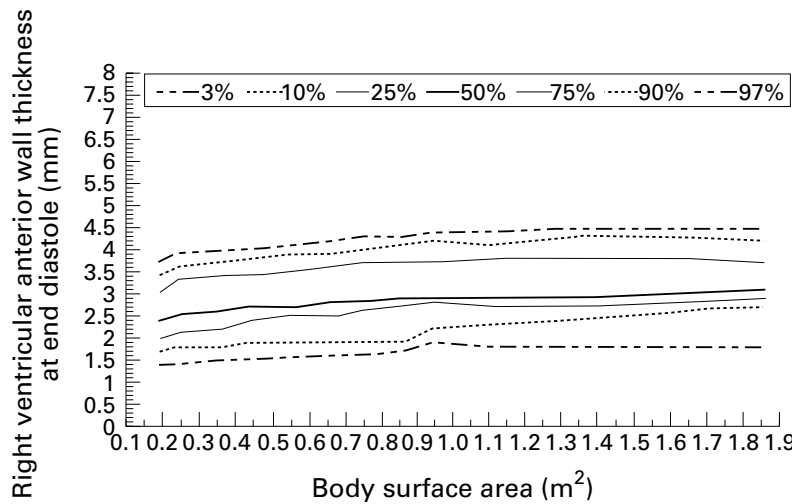

Figure 1 Right ventricular anterior wall thickness at end diastole plotted against the body surface area in 2036 healthy children.

Keywords: paediatric cardiology; echocardiography; normal values

Echocardiography is widely used to diagnose congenital heart defects or exclude cardiac involvement in infectious, neuromuscular, or metabolic disorders. $M$ mode (one dimensional) echocardiography makes it possible to assess and quantitate non-invasively certain aspects of cardiac anatomy and function. However, nearly all published normal values either date from the start of routine echocardiography in the USA (mid-1970s to mid-1980s), or consist of small and heterogeneous samples of healthy infants, children, and young adults. ${ }^{1-6}$ The largest paediatric cohort consisted of 205 healthy children. ${ }^{1}$ The range of suggested normal limits in that study was so narrow that many of the participating children without heart disease had echocardiographic measurements outside of the previously established limits; thus re-evaluation was necessary (on a much smaller study group) to extend the normal limits. ${ }^{4}$ The wide range of changes during the normal development of the cardiac structures requires a large study group for accurate data on normal values, even when centile charts are used. Small step centile charts, which are widely used in paediatrics, have not been available until now for routine echocardiographic measurements.

In the last 15 to 20 years there have also been dramatic improvements in echocardiographic equipment, so that it is now possible to obtain direct digital measurements, while better two dimensional resolution affords more accurate placement of the $M$ mode beam.

Our aim in this study was to re-evaluate the limits of echocardiographic measurements of

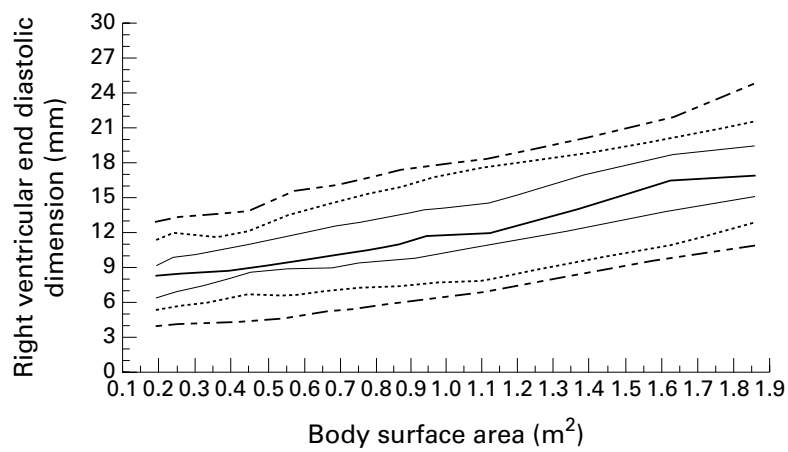

Figure 2 Right ventricular end diastolic dimension plotted against the body surface area in 2036 healthy children. See fig 1 for key. 


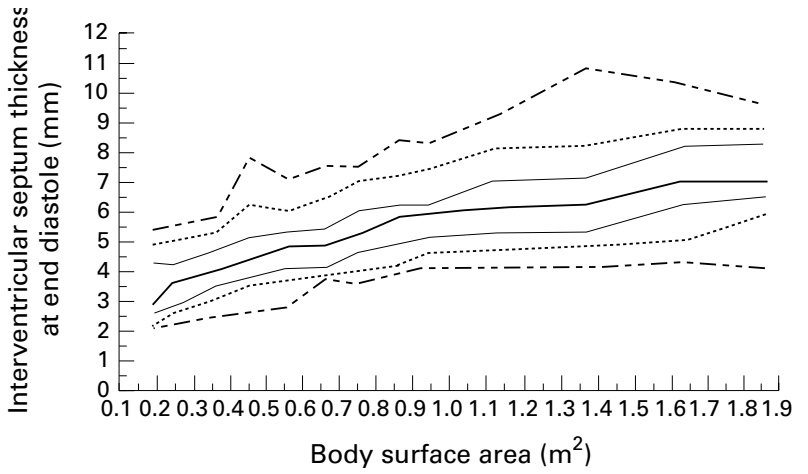

Figure 3 Interventricular septal thickness at end diastole plotted against the body surface area in 2036 healthy children. See fig 1 for key.

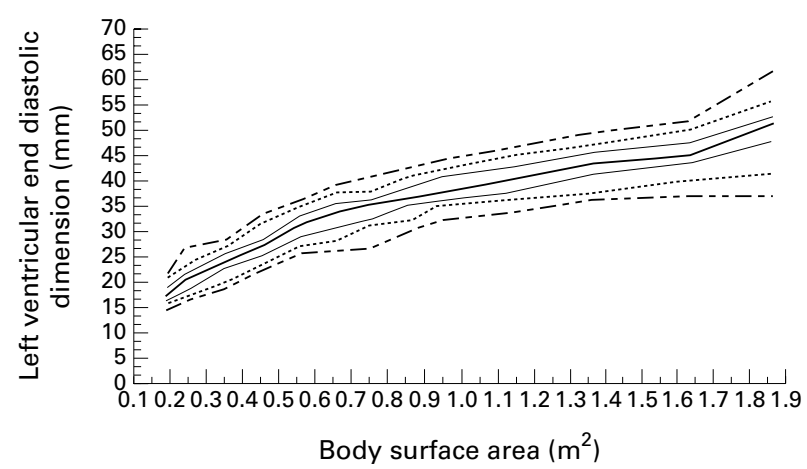

Figure 5 Left ventricular end diastolic dimension plotted against the body surface area in 2036 healthy children. See fig 1 for key.

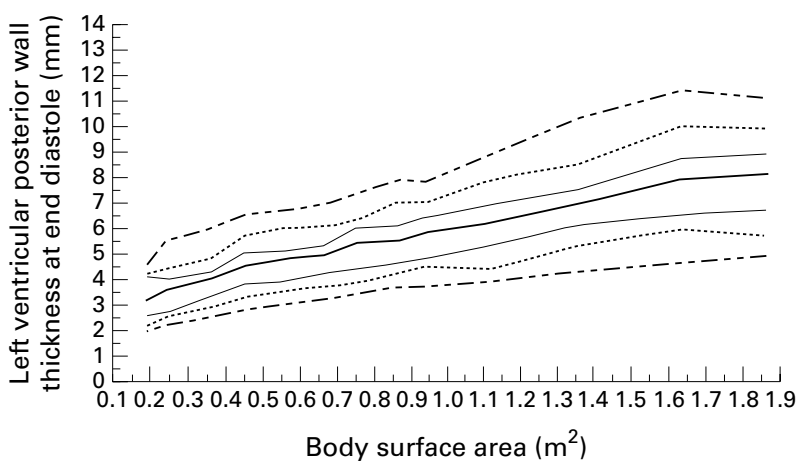

Figure 7 Left ventricular posterior wall thickness at end diastole plotted against the body surface area in 2036 healthy children. See fig 1 for key.

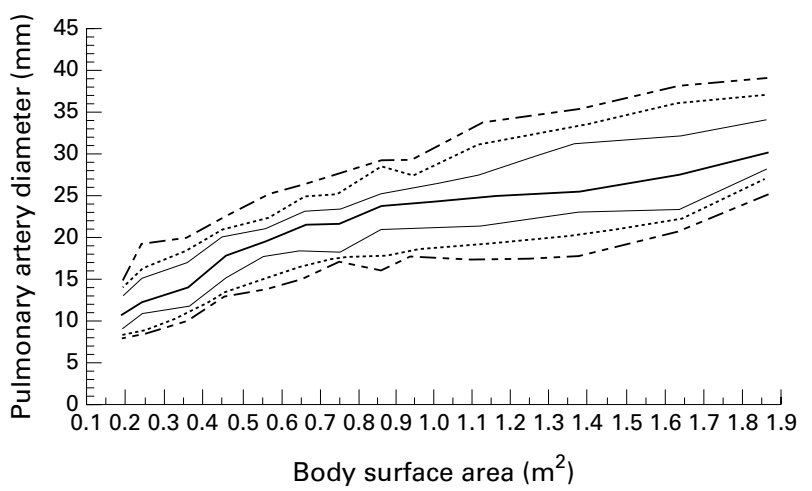

Figure 9 Pulmonary artery diameter plotted against the body surface area in 2036 healthy children. See fig 1 for key.

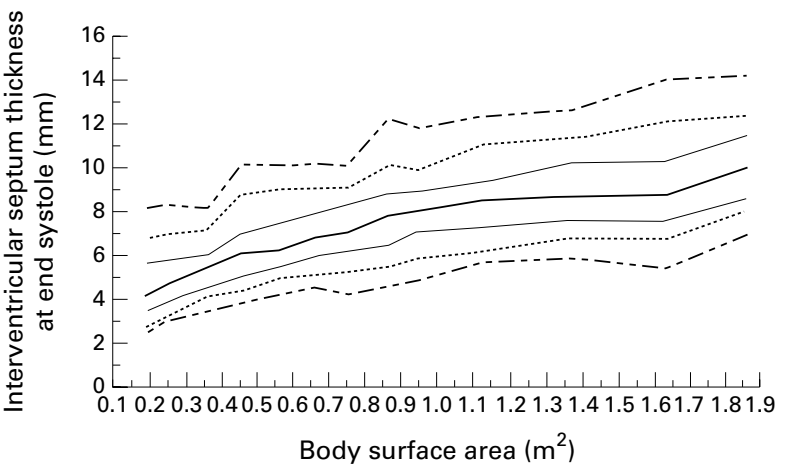

Figure 4 Interventricular septal thickness at end systole plotted against the body surface area in 2036 healthy children. See fig 1 for key.

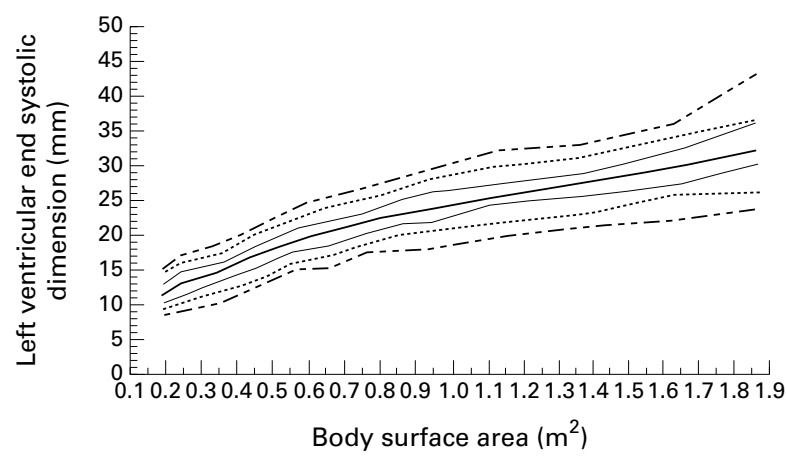

Figure 6 Left ventricular end systolic dimension plotted against the body surface area in 2036 healthy children. See fig 1 for key.

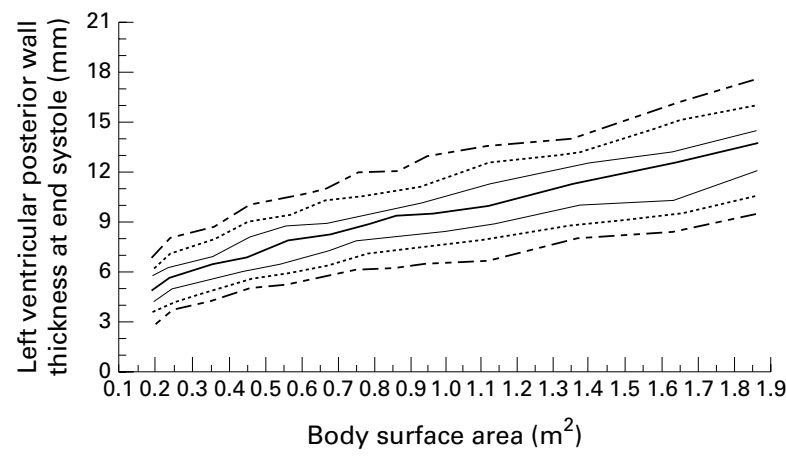

Figure 8 Left ventricular posterior wall thickness at end systole plotted against the body surface area in 2036 healthy children. See fig 1 for key.

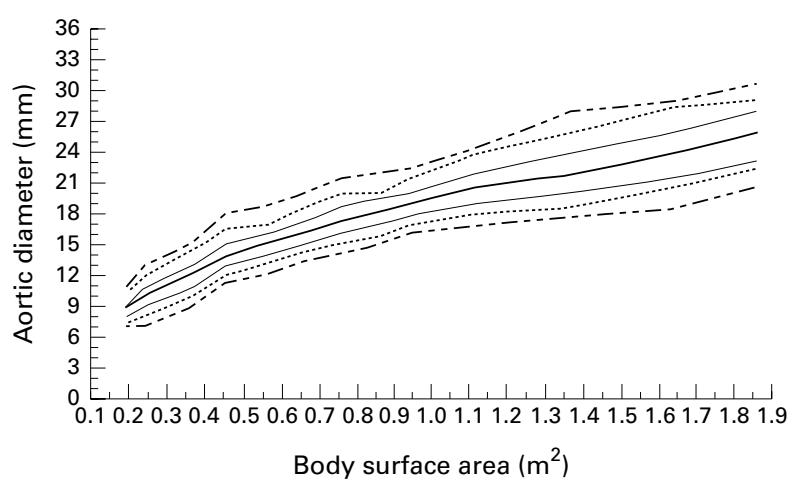

Figure 10 Aortic diameter plotted against the body surface area in 2036 healthy children. See fig 1 for key. 


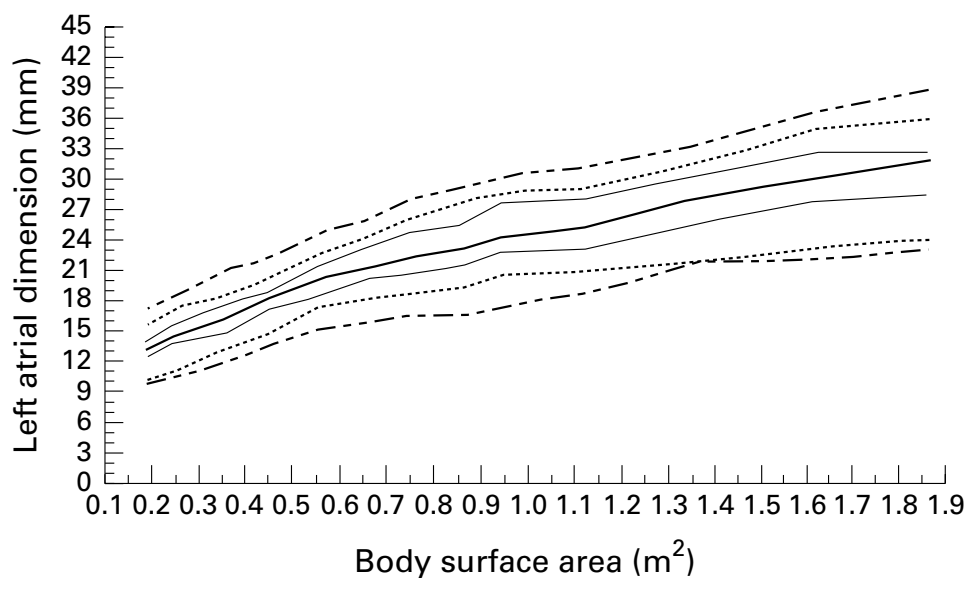

Figure 11 Left atrial dimension plotted against the body surface area in 2036 healthy children. See fig 1 for key.

the normal paediatric population and to develop centile charts.

\section{Methods}

We examined 2036 normal healthy midEuropean newborns, infants, children, and young adults without cardiac heart disease or a history of cardiac involvement in infectious, neuromuscular, or metabolic disorders. Most were outpatients referred for evaluation of a heart murmur which was found to be innocent on clinical, electrocardiographic, and radiological grounds. The others, especially the newborn infants, were inpatients in whom cardiac examination was performed to exclude congenital malformations. There were also 349 older school children, who were volunteers.

No sedation was used during echocardiography. If the children were too restless their measurements were excluded. Phonocardiograms were performed in all patients with heart murmurs, to exclude fixed splitting of heart sounds and pathological murmurs. Chest $x$ rays were only performed in children in whom there was suspicion of a thoracic structural abnormality or a history of chronic obstructive lung disease. Only patients with no evidence of an abnormality were included.
Echocardiographic measurements were made by six different paediatric cardiologists. On the basis of the data of Rogé and colleagues, ${ }^{4}$ we chose to express echocardiographic dimensions in newborn infants in relation to body weight, because when body weight changes from 2 to $4 \mathrm{~kg}$, body surface area changes only minimally. Otherwise, the echocardiographic data were expressed in relation to body surface area. Body surface area was calculated from the relation between body weight and crown-heel length or height, according to the formula of DuBois and DuBois.

The children were examined in the supine position with the right shoulder slightly raised, using modern digital echocardiographic equipment (Toshiba SSA 380 Power-Vision, Tokyo, Japan). Instantaneous measurements were made over three cardiac cycles and the mean values obtained.

Appropriate transducers $(7.5,5.0,3.5$, and 2.5 MHz) were used to define the cardiac structures. The echocardiograms were obtained in the standard precordial positions. ${ }^{48}$ We followed recommendations for standardising measurements made from $M$ mode echocardiograms, as described elsewhere. ${ }^{4910}$

The following measurements were obtained from each subject: right ventricular anterior wall thickness at end diastole (RVAWd) ${ }^{10}$; right ventricular end diastolic dimension (RVDD) ${ }^{10}$; thickness of the interventricular septum at end diastole (IVSd) and end systole (IVSs); left ventricular dimension at end diastole (LVEDD) and end systole (LVESD); left ventricular posterior wall thickness at end diastole (LVPWd) and end systole (LVPWs); pulmonary and aortic diameter ( $\mathrm{PAD}^{4}$ and $\left.\mathrm{AOD}\right)$; and left atrial dimension (LAD). RVAWd and RVDD were measured at the onset of the QRS complex in a plane passing through standard portions of the left ventricle. ${ }^{10}$

Centile charts (3rd, 10th, 25th, 50th, 75th, 90th, and 97th centiles) were constructed for each measurement in relation to body surface area. In newborn infants, mean values with standard deviations are presented in relation to

Table 1 Normal $M$ mode echocardiographic values for neonates with mean body weight between $2000 \mathrm{~g}$ and $4000 \mathrm{~g}$

\begin{tabular}{|c|c|c|c|c|c|c|c|c|c|c|c|}
\hline$B W(k g)$ & $\begin{array}{l}\text { RVAWd } \\
(\mathrm{mm})\end{array}$ & $\begin{array}{l}R V D D \\
(\mathrm{~mm})\end{array}$ & $\begin{array}{l}I V S d \\
(\mathrm{~mm})\end{array}$ & $\begin{array}{l}I V S s \\
(\mathrm{~mm})\end{array}$ & $\begin{array}{l}\text { LVEDD } \\
(\mathrm{mm})\end{array}$ & $\begin{array}{l}\text { LVESD } \\
(\mathrm{mm})\end{array}$ & $\begin{array}{l}\text { LVPWd } \\
(\mathrm{mm})\end{array}$ & $\begin{array}{l}\text { LVPWs } \\
(\mathrm{mm})\end{array}$ & $P A D(\mathrm{~mm})$ & $A o D(m m)$ & $L A D(\mathrm{~mm})$ \\
\hline 2.0 & $\begin{array}{l}1.3 \\
2.4 \\
3.5\end{array}$ & $\begin{array}{r}4.0 \\
\mathbf{8 . 4} \\
12.8\end{array}$ & $\begin{array}{l}2.1 \\
3.5 \\
4.7\end{array}$ & $\begin{array}{l}2.4 \\
4.4 \\
6.4\end{array}$ & $\begin{array}{l}15.0 \\
\mathbf{1 7 . 1} \\
19.2\end{array}$ & $\begin{array}{r}9.7 \\
\mathbf{1 1 . 0} \\
12.3\end{array}$ & $\begin{array}{l}1.9 \\
2.7 \\
3.5\end{array}$ & $\begin{array}{l}2.8 \\
4.5 \\
6.2\end{array}$ & $\begin{array}{r}6.2 \\
\mathbf{9 . 3} \\
12.4\end{array}$ & $\begin{array}{l}6.9 \\
\mathbf{8 . 2} \\
9.5\end{array}$ & $\begin{array}{r}8.3 \\
11.5 \\
14.7\end{array}$ \\
\hline 2.5 & $\begin{array}{l}1.4 \\
2.5 \\
3.6\end{array}$ & $\begin{array}{r}4.0 \\
\mathbf{8 . 4} \\
12.8\end{array}$ & $\begin{array}{l}2.1 \\
3.5 \\
4.7\end{array}$ & $\begin{array}{l}2.4 \\
\mathbf{5 . 0} \\
7.6\end{array}$ & $\begin{array}{l}15.0 \\
\mathbf{1 8 . 1} \\
21.1\end{array}$ & $\begin{array}{r}9.2 \\
\mathbf{1 1 . 7} \\
14.2\end{array}$ & $\begin{array}{l}2.2 \\
\mathbf{3 . 2} \\
4.2\end{array}$ & $\begin{array}{l}2.9 \\
\mathbf{5 . 0} \\
7.1\end{array}$ & $\begin{array}{r}6.8 \\
11.0 \\
15.2\end{array}$ & $\begin{array}{r}7.4 \\
\mathbf{8 . 8} \\
10.2\end{array}$ & $\begin{array}{r}8.5 \\
12.1 \\
15.6\end{array}$ \\
\hline 3.0 & $\begin{array}{l}1.4 \\
2.5 \\
3.6\end{array}$ & $\begin{array}{r}4.1 \\
8.5 \\
12.9\end{array}$ & $\begin{array}{l}2.3 \\
\mathbf{3 . 6} \\
4.9\end{array}$ & $\begin{array}{l}2.5 \\
5.1 \\
7.7\end{array}$ & $\begin{array}{l}15.1 \\
\mathbf{1 8 . 2} \\
21.3\end{array}$ & $\begin{array}{r}9.2 \\
11.7 \\
14.2\end{array}$ & $\begin{array}{l}2.4 \\
3.5 \\
4.6\end{array}$ & $\begin{array}{l}3.1 \\
5.1 \\
7.1\end{array}$ & $\begin{array}{r}7.0 \\
11.0 \\
15.0\end{array}$ & $\begin{array}{r}7.5 \\
\mathbf{9 . 1} \\
10.7\end{array}$ & $\begin{array}{r}9.4 \\
12.6 \\
15.8\end{array}$ \\
\hline 3.5 & $\begin{array}{l}1.5 \\
2.6 \\
3.7\end{array}$ & $\begin{array}{r}4.1 \\
8.6 \\
13.1\end{array}$ & $\begin{array}{l}2.3 \\
3.7 \\
5.1\end{array}$ & $\begin{array}{l}2.5 \\
5.3 \\
8.1\end{array}$ & $\begin{array}{l}15.4 \\
18.8 \\
22.2\end{array}$ & $\begin{array}{r}9.5 \\
\mathbf{1 1 . 9} \\
14.3\end{array}$ & $\begin{array}{l}2.5 \\
3.6 \\
4.7\end{array}$ & $\begin{array}{l}3.3 \\
5.4 \\
7.5\end{array}$ & $\begin{array}{r}8.0 \\
11.2 \\
14.4\end{array}$ & $\begin{array}{r}7.5 \\
\mathbf{9 . 3} \\
11.1\end{array}$ & $\begin{array}{l}10.2 \\
13.2 \\
16.2\end{array}$ \\
\hline 4.0 & $\begin{array}{l}1.5 \\
2.6 \\
3.7\end{array}$ & $\begin{array}{r}4.1 \\
8.6 \\
13.1\end{array}$ & $\begin{array}{l}2.4 \\
3.8 \\
5.2\end{array}$ & $\begin{array}{l}2.6 \\
5.4 \\
8.2\end{array}$ & $\begin{array}{l}16.5 \\
19.9 \\
23.3\end{array}$ & $\begin{array}{l}10.2 \\
\mathbf{1 2 . 7} \\
15.2\end{array}$ & $\begin{array}{l}2.6 \\
\mathbf{3 . 7} \\
4.8\end{array}$ & $\begin{array}{l}3.5 \\
5.7 \\
7.9\end{array}$ & $\begin{array}{r}9.3 \\
12.5 \\
15.7\end{array}$ & $\begin{array}{r}7.6 \\
9.6 \\
11.6\end{array}$ & $\begin{array}{l}10.5 \\
13.7 \\
16.9\end{array}$ \\
\hline
\end{tabular}

The mean value is bold; the value above is mean $-2 \mathrm{SD}$, the value below is mean $+2 \mathrm{SD}$.

AoD, aortic diameter; BW, body weight; IVSd, thickness of interventricular septum at end diastole; IVSs, thickness of interventricular septum at end systole; LAD, left atrial dimension; LVEDD, left ventricular end diastolic dimension; LVESD, left ventricular end systolic dimension; LVPWd, left ventricular posterior wall thickness at end diastole; LVPWs, left ventricular posterior wall thickness at end systole; PAD, pulmonary artery diameter; RVAWd, right ventricular anterior wall thickness at end diastole; RVDD, right ventricular end diastolic dimension. 
body weight for mean weights of between 2 and $4 \mathrm{~kg}$, and in relation to body surface area for areas between 0.25 and $2 \mathrm{~m}^{2}$. For establishing significance, each body weight or surface area group consisted of a minimum of 90 individuals from whom all variables were available.

Statistical analyses and the calculations were performed using SPSS for Windows, version 8.0. Best fit regression equations for each variable were calculated for the 50th centile in relation to body surface area.

\section{Results}

The results are presented graphically as centile charts (figs 1 to 11 ), and in tabular form as means and standard deviations in relation to specific mean body weight and mean body surface area (tables 1 and 2). For clarity in the centile charts, individual data points are omitted. The centile lines are not smoothed, but reflect the centiles of each body surface area group. Multiple correlation coefficients $(r)$ of the $50 \%$ centile related to body surface area for

Table 2 Normal $M$ mode echocardiographic values from infancy to 18 years with a mean body surface area (BSA) from $0.25 \mathrm{~m}^{2}$ to $2.0 \mathrm{~m}^{2}$

\begin{tabular}{|c|c|c|c|c|c|c|c|c|c|c|c|}
\hline$B S A\left(m^{2}\right)$ & $\begin{array}{l}\text { RVAWd } \\
(\mathrm{mm})\end{array}$ & $\begin{array}{l}R V D D \\
(\mathrm{~mm})\end{array}$ & $I V S d(\mathrm{~mm})$ & $I V S s(\mathrm{~mm})$ & $\begin{array}{l}\text { LVEDD } \\
\text { (mm) }\end{array}$ & $\begin{array}{l}\text { LVESD } \\
(\mathrm{mm})\end{array}$ & $\begin{array}{l}\text { LVPWd } \\
(\mathrm{mm})\end{array}$ & $\begin{array}{l}\text { LVPWs } \\
(\mathrm{mm})\end{array}$ & $P A D(\mathrm{~mm})$ & $A o D(\mathrm{~mm})$ & $L A D(\mathrm{~mm})$ \\
\hline 0.25 & $\begin{array}{l}1.4 \\
2.6 \\
3.8\end{array}$ & $\begin{array}{r}4.2 \\
\mathbf{8 . 7} \\
13.2\end{array}$ & $\begin{array}{l}2.4 \\
3.8 \\
5.2\end{array}$ & $\begin{array}{l}2.5 \\
5.2 \\
7.9\end{array}$ & $\begin{array}{l}16.4 \\
\mathbf{2 0 . 0} \\
23.6\end{array}$ & $\begin{array}{l}10.2 \\
\mathbf{1 3 . 2} \\
16.2\end{array}$ & $\begin{array}{l}2.6 \\
3.6 \\
4.6\end{array}$ & $\begin{array}{l}3.7 \\
5.7 \\
7.7\end{array}$ & $\begin{array}{r}9.6 \\
\mathbf{1 2 . 8} \\
16.0\end{array}$ & $\begin{array}{r}8.0 \\
10.4 \\
12.8\end{array}$ & $\begin{array}{l}10.5 \\
\mathbf{1 4 . 0} \\
17.5\end{array}$ \\
\hline 0.275 & $\begin{array}{l}1.4 \\
2.6 \\
3.8\end{array}$ & $\begin{array}{r}4.2 \\
8.7 \\
13.2\end{array}$ & $\begin{array}{l}2.4 \\
3.8 \\
5.2\end{array}$ & $\begin{array}{l}2.6 \\
5.4 \\
8.2\end{array}$ & $\begin{array}{l}17.0 \\
21.2 \\
25.4\end{array}$ & $\begin{array}{l}10.4 \\
13.6 \\
16.8\end{array}$ & $\begin{array}{l}2.7 \\
3.8 \\
4.9\end{array}$ & $\begin{array}{l}3.9 \\
5.9 \\
7.9\end{array}$ & $\begin{array}{r}9.6 \\
\mathbf{1 3 . 6} \\
17.6\end{array}$ & $\begin{array}{r}8.6 \\
11.1 \\
13.6\end{array}$ & $\begin{array}{l}11.5 \\
15.1 \\
18.7\end{array}$ \\
\hline 0.30 & $\begin{array}{l}1.6 \\
2.7 \\
3.8\end{array}$ & $\begin{array}{r}4.2 \\
\mathbf{8 . 7} \\
13.2\end{array}$ & $\begin{array}{l}2.5 \\
3.9 \\
5.3\end{array}$ & $\begin{array}{l}3.0 \\
5.8 \\
8.6\end{array}$ & $\begin{array}{l}18.0 \\
22.9 \\
25.8\end{array}$ & $\begin{array}{l}10.8 \\
\mathbf{1 4 . 8} \\
18.8\end{array}$ & $\begin{array}{l}2.8 \\
4.1 \\
5.4\end{array}$ & $\begin{array}{l}4.2 \\
\mathbf{6 . 3} \\
8.4\end{array}$ & $\begin{array}{l}10.3 \\
14.5 \\
18.7\end{array}$ & $\begin{array}{r}9.0 \\
11.3 \\
13.6\end{array}$ & $\begin{array}{l}11.5 \\
\mathbf{1 5 . 3} \\
19.1\end{array}$ \\
\hline 0.35 & $\begin{array}{l}1.6 \\
2.7 \\
3.8\end{array}$ & $\begin{array}{r}4.3 \\
\mathbf{8 . 8} \\
13.3\end{array}$ & $\begin{array}{l}2.5 \\
3.9 \\
5.3\end{array}$ & $\begin{array}{l}3.0 \\
5.8 \\
8.6\end{array}$ & $\begin{array}{l}19.0 \\
23.6 \\
27.2\end{array}$ & $\begin{array}{l}10.8 \\
14.8 \\
18.8\end{array}$ & $\begin{array}{l}2.8 \\
4.1 \\
5.4\end{array}$ & $\begin{array}{l}4.4 \\
\mathbf{6 . 6} \\
8.8\end{array}$ & $\begin{array}{l}11.0 \\
15.0 \\
19.0\end{array}$ & $\begin{array}{l}10.0 \\
12.0 \\
14.0\end{array}$ & $\begin{array}{l}12.0 \\
16.3 \\
20.6\end{array}$ \\
\hline 0.40 & $\begin{array}{l}1.6 \\
2.7 \\
3.8\end{array}$ & $\begin{array}{r}4.4 \\
8.9 \\
13.4\end{array}$ & $\begin{array}{l}2.6 \\
4.1 \\
5.6\end{array}$ & $\begin{array}{l}3.2 \\
6.2 \\
9.2\end{array}$ & $\begin{array}{l}21.0 \\
\mathbf{2 6 . 0} \\
31.0\end{array}$ & $\begin{array}{l}12.0 \\
\mathbf{1 6 . 1} \\
20.1\end{array}$ & $\begin{array}{l}2.9 \\
4.2 \\
5.5\end{array}$ & $\begin{array}{l}4.5 \\
\mathbf{6 . 8} \\
9.1\end{array}$ & $\begin{array}{l}11.5 \\
\mathbf{1 5 . 4} \\
19.3\end{array}$ & $\begin{array}{l}10.9 \\
\mathbf{1 2 . 9} \\
14.9\end{array}$ & $\begin{array}{l}13.0 \\
16.8 \\
20.6\end{array}$ \\
\hline 0.45 & $\begin{array}{l}1.65 \\
2.75 \\
3.85\end{array}$ & $\begin{array}{r}4.5 \\
\mathbf{9 . 0} \\
13.5\end{array}$ & $\begin{array}{l}2.6 \\
4.2 \\
5.8\end{array}$ & $\begin{array}{l}3.3 \\
\mathbf{6 . 3} \\
9.3\end{array}$ & $\begin{array}{l}22.0 \\
27.1 \\
32.1\end{array}$ & $\begin{array}{l}13.0 \\
\mathbf{1 7 . 0} \\
21.0\end{array}$ & $\begin{array}{l}3.1 \\
4.6 \\
6.1\end{array}$ & $\begin{array}{l}5.0 \\
7.3 \\
9.6\end{array}$ & $\begin{array}{l}12.8 \\
\mathbf{1 7 . 2} \\
21.6\end{array}$ & $\begin{array}{l}11.9 \\
14.1 \\
16.3\end{array}$ & $\begin{array}{l}13.8 \\
\mathbf{1 7 . 8} \\
21.8\end{array}$ \\
\hline 0.50 & $\begin{array}{l}1.65 \\
2.75 \\
3.85\end{array}$ & $\begin{array}{r}4.8 \\
\mathbf{9 . 3} \\
13.8\end{array}$ & $\begin{array}{l}2.7 \\
4.3 \\
5.9\end{array}$ & $\begin{array}{l}3.5 \\
\mathbf{6 . 6} \\
9.7\end{array}$ & $\begin{array}{l}23.4 \\
\mathbf{2 9 . 0} \\
34.6\end{array}$ & $\begin{array}{l}14.0 \\
18.0 \\
22.0\end{array}$ & $\begin{array}{l}3.1 \\
4.6 \\
6.1\end{array}$ & $\begin{array}{l}5.2 \\
7.5 \\
9.8\end{array}$ & $\begin{array}{l}13.6 \\
\mathbf{1 8 . 3} \\
23.0\end{array}$ & $\begin{array}{l}12.2 \\
14.9 \\
17.7\end{array}$ & $\begin{array}{l}14.5 \\
18.7 \\
22.9\end{array}$ \\
\hline 0.55 & $\begin{array}{l}1.65 \\
2.75 \\
3.85\end{array}$ & $\begin{array}{r}5.0 \\
9.5 \\
14.0\end{array}$ & $\begin{array}{l}3.1 \\
4.6 \\
6.1\end{array}$ & $\begin{array}{l}3.7 \\
\mathbf{6 . 8} \\
9.9\end{array}$ & $\begin{array}{l}25.6 \\
31.0 \\
36.4\end{array}$ & $\begin{array}{l}15.0 \\
19.3 \\
23.6\end{array}$ & $\begin{array}{l}3.3 \\
4.8 \\
6.3\end{array}$ & $\begin{array}{r}5.7 \\
\mathbf{8 . 0} \\
10.3\end{array}$ & $\begin{array}{l}14.6 \\
19.6 \\
24.6\end{array}$ & $\begin{array}{l}12.6 \\
15.2 \\
17.8\end{array}$ & $\begin{array}{l}15.3 \\
19.7 \\
24.1\end{array}$ \\
\hline 0.60 & $\begin{array}{l}1.7 \\
\mathbf{2 . 8} \\
3.9\end{array}$ & $\begin{array}{r}5.2 \\
9.6 \\
14.0\end{array}$ & $\begin{array}{l}3.3 \\
4.8 \\
6.3\end{array}$ & $\begin{array}{r}3.8 \\
\mathbf{6 . 9} \\
10.0\end{array}$ & $\begin{array}{l}26.0 \\
31.6 \\
37.2\end{array}$ & $\begin{array}{l}15.4 \\
19.9 \\
24.4\end{array}$ & $\begin{array}{l}3.3 \\
4.8 \\
6.3\end{array}$ & $\begin{array}{r}5.7 \\
\mathbf{8 . 0} \\
10.3\end{array}$ & $\begin{array}{l}15.3 \\
\mathbf{2 0 . 3} \\
25.3\end{array}$ & $\begin{array}{l}12.8 \\
15.6 \\
18.4\end{array}$ & $\begin{array}{l}16.1 \\
20.1 \\
24.1\end{array}$ \\
\hline 0.65 & $\begin{array}{l}1.7 \\
2.8 \\
3.9\end{array}$ & $\begin{array}{r}5.5 \\
9.9 \\
14.3\end{array}$ & $\begin{array}{l}3.3 \\
4.8 \\
6.3\end{array}$ & $\begin{array}{r}3.8 \\
\mathbf{6 . 9} \\
10.0\end{array}$ & $\begin{array}{l}27.2 \\
33.2 \\
39.2\end{array}$ & $\begin{array}{l}15.7 \\
20.4 \\
25.1\end{array}$ & $\begin{array}{l}3.4 \\
4.9 \\
6.4\end{array}$ & $\begin{array}{r}5.8 \\
\mathbf{8 . 2} \\
10.6\end{array}$ & $\begin{array}{l}15.4 \\
20.4 \\
25.4\end{array}$ & $\begin{array}{l}13.2 \\
\mathbf{1 6 . 2} \\
19.2\end{array}$ & $\begin{array}{l}16.1 \\
20.8 \\
25.5\end{array}$ \\
\hline 0.70 & $\begin{array}{l}1.7 \\
\mathbf{2 . 8} \\
3.9\end{array}$ & $\begin{array}{r}5.7 \\
10.1 \\
14.5\end{array}$ & $\begin{array}{l}3.5 \\
5.0 \\
6.5\end{array}$ & $\begin{array}{r}4.2 \\
7.2 \\
10.2\end{array}$ & $\begin{array}{l}27.4 \\
33.9 \\
40.4\end{array}$ & $\begin{array}{l}16.1 \\
21.3 \\
26.5\end{array}$ & $\begin{array}{l}3.5 \\
5.2 \\
6.9\end{array}$ & $\begin{array}{r}6.1 \\
8.7 \\
11.3\end{array}$ & $\begin{array}{l}15.8 \\
\mathbf{2 0 . 8} \\
25.8\end{array}$ & $\begin{array}{l}13.5 \\
16.9 \\
20.3\end{array}$ & $\begin{array}{l}16.2 \\
21.2 \\
26.2\end{array}$ \\
\hline 0.80 & $\begin{array}{l}1.7 \\
\mathbf{2 . 8} \\
3.9\end{array}$ & $\begin{array}{r}5.8 \\
\mathbf{1 0 . 5} \\
15.2\end{array}$ & $\begin{array}{l}3.6 \\
5.2 \\
6.8\end{array}$ & $\begin{array}{r}4.4 \\
7.5 \\
10.6\end{array}$ & $\begin{array}{l}29.6 \\
35.8 \\
42.0\end{array}$ & $\begin{array}{l}17.7 \\
\mathbf{2 2 . 7} \\
27.7\end{array}$ & $\begin{array}{l}3.6 \\
\mathbf{5 . 7} \\
7.8\end{array}$ & $\begin{array}{r}6.2 \\
\mathbf{9 . 1} \\
12.0\end{array}$ & $\begin{array}{l}15.8 \\
\mathbf{2 0 . 8} \\
25.8\end{array}$ & $\begin{array}{l}14.5 \\
17.9 \\
21.3\end{array}$ & $\begin{array}{l}16.5 \\
22.5 \\
28.5\end{array}$ \\
\hline 0.90 & $\begin{array}{l}1.7 \\
2.8 \\
3.9\end{array}$ & $\begin{array}{r}6.4 \\
\mathbf{1 1 . 0} \\
15.6\end{array}$ & $\begin{array}{l}3.8 \\
5.6 \\
7.4\end{array}$ & $\begin{array}{r}4.9 \\
8.3 \\
11.7\end{array}$ & $\begin{array}{l}31.0 \\
37.1 \\
43.2\end{array}$ & $\begin{array}{l}18.0 \\
\mathbf{2 3 . 6} \\
29.2\end{array}$ & $\begin{array}{l}3.7 \\
5.9 \\
8.1\end{array}$ & $\begin{array}{r}6.8 \\
\mathbf{9 . 5} \\
12.2\end{array}$ & $\begin{array}{l}16.7 \\
22.5 \\
28.3\end{array}$ & $\begin{array}{l}15.1 \\
18.7 \\
22.3\end{array}$ & $\begin{array}{l}17.0 \\
23.2 \\
29.4\end{array}$ \\
\hline 1.00 & $\begin{array}{l}1.7 \\
\mathbf{2 . 8} \\
3.9\end{array}$ & $\begin{array}{r}6.4 \\
11.2 \\
16.0\end{array}$ & $\begin{array}{l}4.0 \\
5.8 \\
7.6\end{array}$ & $\begin{array}{r}5.1 \\
8.4 \\
11.7\end{array}$ & $\begin{array}{l}31.7 \\
38.5 \\
45.3\end{array}$ & $\begin{array}{l}18.6 \\
\mathbf{2 4 . 4} \\
30.2\end{array}$ & $\begin{array}{l}3.7 \\
5.9 \\
8.1\end{array}$ & $\begin{array}{r}6.8 \\
\mathbf{9 . 5} \\
12.2\end{array}$ & $\begin{array}{l}17.8 \\
\mathbf{2 4} \\
30.2\end{array}$ & $\begin{array}{l}16.3 \\
19.9 \\
23.5\end{array}$ & $\begin{array}{l}19.2 \\
\mathbf{2 5} \\
30.8\end{array}$ \\
\hline 1.10 & $\begin{array}{l}1.8 \\
2.9 \\
4.0\end{array}$ & $\begin{array}{r}7.4 \\
11.8 \\
16.2\end{array}$ & $\begin{array}{l}4.3 \\
6.2 \\
8.1\end{array}$ & $\begin{array}{r}5.4 \\
9.0 \\
12.6\end{array}$ & $\begin{array}{l}32.5 \\
39.4 \\
46.3\end{array}$ & $\begin{array}{l}19.6 \\
\mathbf{2 5 . 2} \\
30.8\end{array}$ & $\begin{array}{l}3.9 \\
\mathbf{6 . 3} \\
8.7\end{array}$ & $\begin{array}{r}7.0 \\
\mathbf{1 0 . 3} \\
13.6\end{array}$ & $\begin{array}{l}17.8 \\
\mathbf{2 4} \\
30.2\end{array}$ & $\begin{array}{l}17.5 \\
\mathbf{2 0 . 9} \\
24.3\end{array}$ & $\begin{array}{l}19.5 \\
\mathbf{2 5 . 2} \\
30.9\end{array}$ \\
\hline 1.20 & $\begin{array}{l}1.8 \\
2.9 \\
4.0\end{array}$ & $\begin{array}{r}7.6 \\
\mathbf{1 2 . 4} \\
17.2\end{array}$ & $\begin{array}{l}4.7 \\
6.5 \\
8.3\end{array}$ & $\begin{array}{r}5.4 \\
9.0 \\
12.6\end{array}$ & $\begin{array}{l}35.5 \\
41.7 \\
47.9\end{array}$ & $\begin{array}{l}21.5 \\
27.1 \\
32.7\end{array}$ & $\begin{array}{l}4.0 \\
\mathbf{6 . 6} \\
9.2\end{array}$ & $\begin{array}{r}7.6 \\
\mathbf{1 0 . 7} \\
13.8\end{array}$ & $\begin{array}{l}18.3 \\
\mathbf{2 4 . 3} \\
30.3\end{array}$ & $\begin{array}{l}17.5 \\
21.0 \\
24.5\end{array}$ & $\begin{array}{l}20.9 \\
\mathbf{2 6 . 0} \\
31.1\end{array}$ \\
\hline 1.30 & $\begin{array}{l}1.9 \\
3.0 \\
4.1\end{array}$ & $\begin{array}{r}8.5 \\
13.5 \\
18.5\end{array}$ & $\begin{array}{l}4.8 \\
6.6 \\
8.4\end{array}$ & $\begin{array}{r}5.4 \\
9.0 \\
12.6\end{array}$ & $\begin{array}{l}35.8 \\
\mathbf{4 2 . 4} \\
49.0\end{array}$ & $\begin{array}{l}21.5 \\
27.1 \\
32.7\end{array}$ & $\begin{array}{l}4.3 \\
6.9 \\
9.5\end{array}$ & $\begin{array}{r}8.1 \\
\mathbf{1 1 . 0} \\
13.9\end{array}$ & $\begin{array}{l}18.8 \\
\mathbf{2 4 . 6} \\
30.4\end{array}$ & $\begin{array}{l}17.5 \\
21.7 \\
25.9\end{array}$ & $\begin{array}{l}21.7 \\
\mathbf{2 7 . 3} \\
32.9\end{array}$ \\
\hline 1.40 & $\begin{array}{l}1.9 \\
3.0 \\
4.1\end{array}$ & $\begin{array}{r}9.0 \\
\mathbf{1 4 . 0} \\
19.0\end{array}$ & $\begin{array}{l}4.9 \\
\mathbf{6 . 7} \\
8.5\end{array}$ & $\begin{array}{r}5.8 \\
9.2 \\
12.6\end{array}$ & $\begin{array}{l}37.3 \\
43.3 \\
49.3\end{array}$ & $\begin{array}{l}22.0 \\
\mathbf{2 7 . 6} \\
33.2\end{array}$ & $\begin{array}{l}4.3 \\
\mathbf{6 . 9} \\
9.5\end{array}$ & $\begin{array}{r}8.5 \\
11.5 \\
14.5\end{array}$ & $\begin{array}{l}21.4 \\
\mathbf{2 6 . 8} \\
32.2\end{array}$ & $\begin{array}{l}17.9 \\
22.7 \\
27.5\end{array}$ & $\begin{array}{l}22.8 \\
\mathbf{2 8 . 2} \\
33.6\end{array}$ \\
\hline 1.50 & $\begin{array}{l}1.9 \\
3.1 \\
4.3\end{array}$ & $\begin{array}{l}10.0 \\
15.6 \\
21.2\end{array}$ & $\begin{array}{l}5.2 \\
7.4 \\
9.6\end{array}$ & $\begin{array}{r}5.8 \\
9.5 \\
13.2\end{array}$ & $\begin{array}{l}39.0 \\
45.4 \\
51.8\end{array}$ & $\begin{array}{l}22.5 \\
\mathbf{2 8 . 6} \\
34.7\end{array}$ & $\begin{array}{r}4.9 \\
7.7 \\
10.5\end{array}$ & $\begin{array}{r}8.5 \\
12.0 \\
15.5\end{array}$ & $\begin{array}{l}21.8 \\
\mathbf{2 7 . 4} \\
33.0\end{array}$ & $\begin{array}{l}18.2 \\
\mathbf{2 3 . 6} \\
29.0\end{array}$ & $\begin{array}{l}23.7 \\
\mathbf{2 9 . 9} \\
36.1\end{array}$ \\
\hline 1.75 & $\begin{array}{l}1.9 \\
3.1 \\
4.3\end{array}$ & $\begin{array}{l}10.3 \\
16.5 \\
22.7\end{array}$ & $\begin{array}{r}5.6 \\
\mathbf{8 . 0} \\
10.4\end{array}$ & $\begin{array}{r}5.8 \\
9.8 \\
13.8\end{array}$ & $\begin{array}{l}36.8 \\
46.8 \\
54.8\end{array}$ & $\begin{array}{l}23.4 \\
\mathbf{2 9 . 8} \\
36.2\end{array}$ & $\begin{array}{r}5.1 \\
8.1 \\
11.1\end{array}$ & $\begin{array}{r}9.5 \\
12.8 \\
16.1\end{array}$ & $\begin{array}{l}22.5 \\
28.5 \\
34.5\end{array}$ & $\begin{array}{l}18.2 \\
\mathbf{2 4 . 4} \\
30.6\end{array}$ & $\begin{array}{l}23.8 \\
\mathbf{3 0 . 4} \\
37.0\end{array}$ \\
\hline 2.00 & $\begin{array}{l}1.9 \\
3.1 \\
4.3\end{array}$ & $\begin{array}{l}11.5 \\
17.5 \\
23.5\end{array}$ & $\begin{array}{r}6.8 \\
9.3 \\
11.8\end{array}$ & $\begin{array}{r}6.5 \\
10.3 \\
14.1\end{array}$ & $\begin{array}{l}45.4 \\
\mathbf{5 3 . 4} \\
61.4\end{array}$ & $\begin{array}{l}25.6 \\
34.4 \\
43.2\end{array}$ & $\begin{array}{r}5.1 \\
8.1 \\
11.1\end{array}$ & $\begin{array}{r}9.6 \\
14.2 \\
18.8\end{array}$ & $\begin{array}{l}23.5 \\
29.5 \\
35.5\end{array}$ & $\begin{array}{l}23 \\
27.4 \\
31.8\end{array}$ & $\begin{array}{l}23.7 \\
32.5 \\
41.3\end{array}$ \\
\hline
\end{tabular}


Table 3 Regression equations of the different variables

\begin{tabular}{lcl}
\hline Variable & Regression equation & $r^{2}$ \\
\hline RVAWd $(\mathrm{mm})$ & $2.8857^{\star} \mathrm{BSA}^{0.1015}$ & 0.9668 \\
RVDD $(\mathrm{mm})$ & $7.4461^{\star} \mathrm{e}^{0.4587^{\star} \mathrm{BSA}}$ & 0.9898 \\
IVSd $(\mathrm{mm})$ & $5.8055^{\star} \mathrm{BSA}^{0.3688}$ & 0.9772 \\
IVSs $(\mathrm{mm})$ & $7.9158^{\star} \mathrm{BSA}^{0.3603}$ & 0.9865 \\
LVEDD (mm) & $38.537^{\star} \mathrm{BSA}^{0.4509}$ & 0.9896 \\
LVESD (mm) & $24.231^{\star} \mathrm{BSA}^{0.4469}$ & 0.9972 \\
LVPWd (mm) & $6.0963^{\star} \mathrm{BSA}^{0.3988}$ & 0.9854 \\
LVPWs (mm) & $9.9845^{\star} \mathrm{BSA}^{0.4238}$ & 0.9908 \\
AoD (mm) & $19.443^{\star} \mathrm{BSA}^{0.4578}$ & 0.9981 \\
PAD (mm) & $8.2037^{\star} \mathrm{Ln}^{(\mathrm{BSA})+23.968}$ & 0.9818 \\
LAD (mm) & $24.695^{\star} \mathrm{BSA}^{0.3911}$ & 0.9975 \\
\hline
\end{tabular}

The regression equation were calculated as best fitting regressions to the $50 \%$ centile line. The multiple squared correlation coefficient $\left(r^{2}\right)$ donates the correlation between the regression equation and the $50 \%$ centile.

See table 1 for key to abbreviations.

each variable, and the accompanying regression equations, are shown in table 3.

Because of the dramatic changes of cardiac dimensions during the early years of child development, small increments were chosen between the smaller body surface area groups (0.025-0.05 $\left.\mathrm{m}^{2}\right)$ and larger increments between the larger body surface area groups $\left(0.1-0.25 \mathrm{~m}^{2}\right)$.

\section{Discussion}

The neonates, infants, children, and adolescents taking part in this study represented a homogeneous sample of the normal population. Their heights and weights were all within the normal range on standard growth charts. They were examined by at least two paediatric cardiologists to ensure that they had normal hearts before the echocardiograms were obtained. In all cases their ECG recordings were within age appropriate normal limits. ${ }^{11}$ Phonocardiograms were performed in all patients with a heart murmur to exclude fixed splitting of heart sounds and pathological murmurs. Chest $x$ rays were obtained if there was a suspicion of thoracic dystrophy or a history of chronic obstructive lung disease, and only those with no evidence of abnormality were included. Echocardiographic data were not used to define participation in the study. All the subjects had complete cross sectional, $M$ mode, and Doppler echocardiographic examinations. The population was thus highly screened and considered to be normal.

It has been suggested that the best correlation is obtained between echocardiographic measurements and height or the cube root of weight. ${ }^{12}$ The variables height, weight, body surface area, and cube root of weight all correlate well with each other. ${ }^{4}$ In fact, the strongest correlations are seen with cube root of weight and body surface area, except in newborn infants. We chose body weight in neonates, because an increase in body weight does not lead to a concomitant increase in body surface area at that stage of life. ${ }^{13}$ In older children, it was more practical to relate the measurements to body surface area, because all modern echocardiographic equipment is programmed to calculate body surface area according to the DuBois formula, and because haemodynamic data are usually expressed in relation to surface area. ${ }^{14}$ However, there is a theoretical problem in relating data to body surface area in children with haemodynamically significant congenital cardiac defects. These children often have impaired growth, so that they will be below the normal centiles for age and will tend to be compared with younger, adequately grown children. In these cases, the centile charts can be used for individual follow up or for assessment of remodelling of cardiac structures after corrective surgery.

The centiles presented in the figures are not smoothed to plot a continuous increase in dimensions, but reflect the calculated centiles for each body surface area group. We believe that the influence of systematic errors as statistical noise in large samples increases with smoothing.

It has been suggested that there might be a tendency for sex and race dependency of echocardiographic measurements. ${ }^{15}{ }^{16}$ In our study racial differences were excluded as only white central European children were examined. There were no significant differences in echocardiographic values between the sexes. The reason for this might be that, as there were six different paediatric cardiologists examining the children, a small sex difference could have been within the range of interobserver variability. On the other hand, in studies in which sex differences have been found, the oldest subjects were all much older than 18 years. ${ }^{316}$ It is possible that sex related differences in cardiac structures develop during or after puberty, or according to physical activity as an adult. The age of the participants in our study cohort was limited to 18 years, and most of the older subjects were still at school.

At all ages, even in the younger subjects, there was a substantial difference between aortic and pulmonary diameters, combined with a wider scatter of normal pulmonary diameter values. The aortic root diameter was measured at end diastole, while the pulmonary diameter was measured whenever it was possible. The wider confidence limits of the pulmonary artery dimension confirm that the pulmonary artery has much greater systolic excursion and widening than the aorta in the young. ${ }^{17}$ This has also been shown using cross sectional echocardiographic measurements in normal children from infancy to adulthood. ${ }^{18}$ However, this finding may also reflect poor boundary detection, as the aorta is measured using the machine's axial resolution whereas the pulmonary artery walls are subject to lateral resolution error, even though there is better resolution of cardiac structures in the younger population. Furthermore, there may be a problem of lung echoes obscuring the lateral pulmonary artery wall. In our study, colour Doppler was not used to delineate chamber/vessel boundaries.

In comparison with previously published normal values, the ranges of our centile charts and the mean values with standard deviations are smaller than Rogé's confidence limits ${ }^{4}$ and wider than Epstein's. ${ }^{1}$ The reason for this might be that Rogé extrapolated the confidence limits, while we calculated centile charts. Rogés data can therefore be used to compare population means but not to predict whether a particular patient has normal or abnormal 
echocardiographic measurements. In a recent study it was shown that data obtained from multiple small population studies combined by meta-analysis have a drawback in that they are overly affected by the heterogeneity of the populations examined. ${ }^{19}$ It therefore seemed necessary to establish normal values for a large homogeneous paediatric population. However, the most important reason for establishing normal values and centile charts for echocardiographic dimensions in normal children is to identify quantitative abnormalities.

1 Epstein ML, Goldberg SJ, Allen HD, et al. Great vessel, cardiac chamber and wall growth patterns in normal children. Circulation 1975;51:1124-9.

2 Gutgesell HP, Paquet M, Duff DF, et al. Evaluation of left ventricular size and function by echocardiography. Results in normal children. Circulation 1977;56:457-62.

3 Henry WL, Ware J, Gardin JM, et al. Echocardiographic measurements in normal subjects. Growth related changes measurements in normal subjects. Growth related changes that occur between

4 Rogé CLL, Silverman NH, Hart PA, et al. Cardiac structure growth pattern determined by echocardiography. Circula tion 1978;57:285-90.

5 Henry WL, Gardin JM, Ware JH. Echocardiographic measurements in normal subjects from infancy to old age. Circulation 1980;62:1054-61.

6 Kampmann C. Echokardiografische Normwerte im Kindesalter. In: Schmailzl KJG, ed. Kardiale Ultraschalldiagnostik, Handbuch und Atlas. Berlin: Blackwell Wissenschaft, 1994: 389-91.
7 DuBois D, DuBois EF, A formula to estimate the approximate surface area if height and weight be known. Arch Intern Med 1916;17:863.

8 Popp RL. Echocardiographic assessment of cardiac disease. Circulation 1976;54:538-41.

9 Roeland J, Gibson DG. Recommendations for standardization of measurements from M-mode echocardiograms. Eur Heart 7 1980;1:375-8.

10 Sahn DJ, DeMaria A, Kisslo J, et al. Recommendations regarding quantitation in M-mode echocardiography: results of a survey of echocardiographic measurements. Circulation 1978;58:1072-83.

11 Davignon A, Rautaharjup P, Boisselle E, et al. Normal ECG standards for children. Pediatr Cardiol 1980;1:133-52.

12 Lundström NR. Clinical applications of echocardiography in infants and children. Acta Paediatr Scand 1974;63:23-30.

13 Baylen B, Meyer RA, Korfhagen A, et al. Left ventricular performance in the critically ill premature infant with patent ductus arteriosus and pulmonary disease. Circulation 1977;55:182-6.

14 Gutgesell HP, Rembold CM. Growth of the human heart relative to body surface area. Am 7 Cardiol 1990;65:662-8.

15 Rowlatt UF, Rimoldi HJ, Lev M. The quantitative anatomy of the child's heart. Pediatr Clin North Am 1963;10:499-518.

16 Lester LA, Sodt PC, Hutcheon N, et al. M-mode echocardiography in normal children and adolescents: some new perspectives. Pediatr Cardiol 1987;8:27-33.

17 Sahn DJ. Determination of cardiac output by echocardiographic Doppler methods: relative accuracy of various sites for measurement. F Am Coll Cardiol 1985;6:663-4.

18 Snider AR, Enderlein MA, Teitel DF, et al. Twodimensional echocardiographic determination of aortic and pulmonary artery sizes from infancy to adulthood in normal subjects. Am $\mathcal{F}$ Cardiol 1984;53:218-24.

19 DerSimonian R, Levine RJ. Resolving discrepancies between a meta-analysis and a subsequent large controlled trial. FAMA 1999;282:664-70.

\section{IMAGES IN CARDIOLOGY}

\section{Occlusion of the left main coronary artery without myocardial infarction}

A 75 year old man was admitted because of stable angina pectoris without any history of myocardial infarction. His risk profile consisted of arterial hypertension and hypercholesterolaemia. At the time he was being treated with $100 \mathrm{mg}$ aspirin, $100 \mathrm{mg}$ metoprolol, $20 \mathrm{mg}$ pravastatin, and $40 \mathrm{mg}$ isosorbide mononitrate daily. ECG showed sinus rhythm, no $Q$ waves, and slight $\mathrm{T}$ wave inversions at lead $\mathrm{aVL}$ and $\mathrm{I}$. A bicycle stress test resulted in horizontal ST segment depression of $2 \mathrm{~mm}$ at $75 \mathrm{~W}$. Coronary angiography was performed and revealed coronary artery disease with complete occlusion of the proximal part of the left coronary artery. The left anterior descending and left circumflex (LCX) branch of the left coronary artery were filled by collateral vessels originating from a large right coronary artery. An additional severe stenosis was found in the proximal part of the LCX. The left ventriculogram did not show any wall motion abnormalities with an ejection fraction of $68 \%$. Immediate coronary bypass grafting was performed without any complication.

According to the literature total occlusion of the left main coronary artery usually causes a

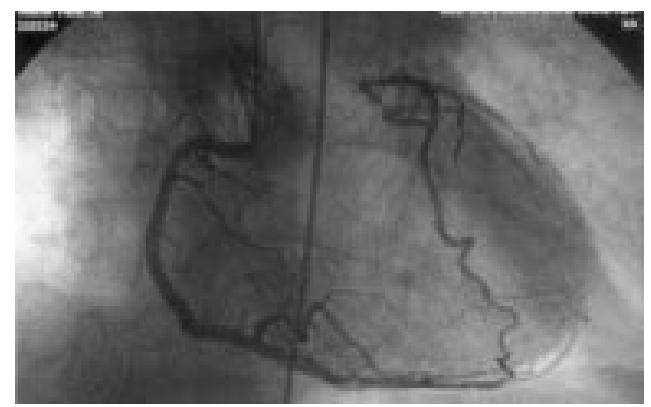

large infarction of the anterior wall leading to cardiogenic shock or sudden cardiac death. Sporadically left main occlusion may occur as a severe complication of percutaneous transluminal balloon angioplasty requiring urgent bypass surgery. This case shows that slow occlusion of the left main coronary artery may develop without myocardial infarction because of excellent collateral blood flow.

ROBERT ZWEIKER KURT STOSCHITZKY WERNER KLEIN 